
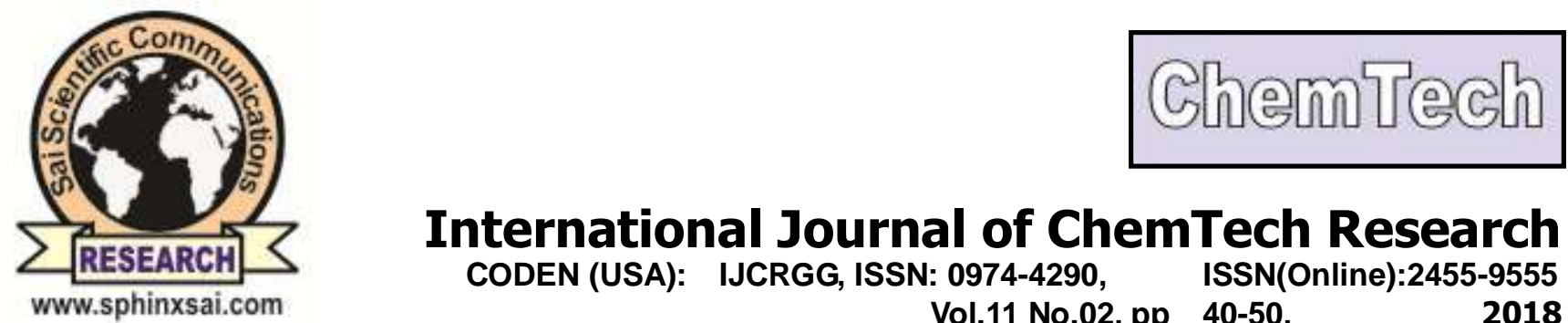

International Journal of ChemTech Research CODEN (USA): IJCRGG, ISSN: 0974-4290, ISSN(Online):2455-9555

Vol.11 No.02, pp 40-50,

2018

\title{
Molecularly Imprinted Polymers as b-sitosterol selective adsorbent using combination of Methacrylate Acid and Trimethylpropane Trimethacrylate
}

\author{
St. Fauziah*1, Nunuk Hariani Soekamto ${ }^{1}$, Muh. Bachri Amran², \\ Paulina Taba ${ }^{1}$ and Prastawa Budi ${ }^{1}$
}

${ }^{1}$ Department of Chemistry, The Faculty of Mathematics and Natural Science, The University of Hasanuddin, Jln. Perintis Kemerdekaan KM. X, Makassar, Indonesia, 90245.

${ }^{2}$ Department of Chemistry, The Faculty of Mathematics and Natural Science, Institute Technology of Bandung, Jl. Ganesa 10 Bandung, Indonesia, 40132

\begin{abstract}
The research aim was to syntheze Molecularly Imprinted Polymers (MIP) that has a memory effect on $\beta$ - sitosterol. The synthesis was conducted through a bulk polymerization process using a methacrylic acid monomer (MAA), trimethyl propane trimethacrylate (TRIM) as a cross linker and $\beta$-sitosterol as a template. The MIP was used as a selective adsorbent of $\beta$-sitosterol. Characterization of MIP was performed using FTIR, SEM, and TGA. The $\beta$ sitosterol adsorption ability of MIP optimized at various $\mathrm{pH}$ and time. The amount of $\beta$ sitosterol adsorbed by MIP was analyzed using HPLC. The FTIR spectrum shows that functional groups that play a role in MIP formation are $\mathrm{OH}, \mathrm{C}=\mathrm{C}$ and $\mathrm{C}=\mathrm{O}$. The MIP morphological shape was spherical like a ball with a particle diameter size of about $2.5 \mu \mathrm{m}$ and the surface of MIP was rougher than NIP. The analysis results of HPLC proven that MIP was able to adsorb $\beta$-sitosterol better than NIP.
\end{abstract}

Key words : Synthesis, MIP, TRIM, MAA, $\beta$-sitosterol, cross-linker.

\section{Introduction}

The separation and purification of a chemical compound from natural product remains an obstacle because the complexity of components in the sample extract. The important step in the separation process was extraction, but it takes a long time and expensive. The best and most efficient way to solve this problem wasa selective extraction to a target compound and important to be separated ${ }^{1}$, however the selective extraction of a compound in the sample requires selective materials in the form of synthetic polymers. The synthetic polymers can be prepared by polymer imprinting techniques through free radical polymerization reactions to produce molecularly imprinting polymers (MIPs) ${ }^{13}$.

International Journal of ChemTech Research, 2018,11(02): 40-50.

DOI= http://dx.doi.org/10.20902/IJCTR.2018.110207 
The MIPs are very stableat various $\mathrm{pH}$, solvent, temperature, and very selective to target molecules ${ }^{6,3}$ and can also be used more than once ${ }^{17}$. Currently, MIP applications are increasing in the fields of chemistry, biology, health and environment ${ }^{11,12}$ especially as adsorbent or stationary phase for the separation compounds, as biosensor and drugmaterial ${ }^{9,11,12}$. In the field of chemistry, MIP was widely used as a material for the separation of amino acid compounds, isomers and enantiomer monomers from natural material samples ${ }^{2}$. The MIP as a selective adsorbent can be produced using appropriate functional monomers and cross-linkers. The monomers are important to determine the ability of MIP to interact selectively with molecules target ${ }^{7}$. Application of appropriate cross-linkers can determine the stability of MIP formed during the polymerization process ${ }^{1}$.

In a previous study, the use of a MAA monomer and an EGDMA cross-linker (MIP_MAA-coEGDMA) has shown better adsorption and stability capabilities ${ }^{10}$ than the MIP using a trifluoro methylacrylic acid (TFMAA) monomer and the EGDMA cross-linker (MIP_TFMAA-co-EGDMA) ${ }^{4}$. The condition was affected by steric and induction effects of the fluorine (F) group on the TFMAA monomer, so that the binding ability between the monomers and the cross-linkers reduces. The MIP used a TRIM cross-linker combined with a TFMAA monomer (MIP_TFMAA-co-TRIM) had a better adsorption capability and were more stable than MIP synthesized using TFMAA monomer combined with EGDMA cross linking (MIP_TFMAA-co-EGDMA). TRIM is a superior cross-linker compared to EGDMA cross-linkers because it has more vinyl clusters which contribute to binding with more monomers so that the resulting polymer is more stable and has a high degree of stiffness ${ }^{14}$.

Therefore, the proper selection of monomers and cross-linkers are necessary. In this study, the template molecules used were $\beta$-sitosterol compounds. The target molecule is one of plant and animal steroid compounds that often interfere in the process of separating and purifying certain compounds in natural products ${ }^{15}$. Therefore $\beta$-sitosterol was selected as a template molecule in MIP synthesis as a selective adsorbent of $\beta$-sitosterol.

\section{Experimental}

\section{Material}

The materials used in the study consisted of methacrylic acid 99\% (Aldrich sigma), $\beta$-sitosterol $97 \%$ (Aldrich sigma), trimethyl propane trimethacrylate 98\% (Aldrich sigma), toluene (E. Merck) as porogen solvent,2, 2 '-azobisisobutironitril (Sigma Aldrich) as the initiator, tetrahydrofurane (THF) (E. Merck) and acetic acid (E. Merck). Double distilled water and methanol (HPLC grade) were used in HPLC analysis. Equipment used include stirrer, glass equipment, digital balance, water bath, oven, HPLC instrument of Agilent 1260 infinity with the column type Cronus RP E18C and column length $12.5 \mathrm{~cm}$ x $0.4 \mathrm{~cm}$, FTIR Shimadzu type IRPrestige-21, and SEM of type Vega 3SB and EDS 6510 (LA, and TGA NETZ.SCH STA 449F.

\section{Synthesis of MIP_MAA-co-TRIM}

In the MIP synthesis, $2 \mathrm{ml}$ of MAA functional monomer and $0.05 \mathrm{~g}$ of $\beta$-sitosterol were dissolved in a round bottom flask containing $10 \mathrm{ml}(9.41 \mathrm{mmol})$ of toluene. After being dissolved, $3 \mathrm{ml}(9.3 \mathrm{mmol})$ of TRIM and $0.05 \mathrm{~g}(0.3 \mathrm{mmol})$ AIBN were added. The solution was sonicated and nitrogen gas was flowed into the solution to remove dissolved oxygen. The polymerization process was carried out in the water bath at $55^{\circ} \mathrm{C}$ for 24 hours. The synthesized polymer was dried, and then washed with THF, methanol/acetic acid (9:1), and double distilled water to remove the template, porogenous solvent and other compounds. The washed polymer was dried at $50^{\circ} \mathrm{C}$. The Non-imprinting polymer (NIP) was made without the molecular template with the same synthesis procedure as $\mathrm{MIP}^{13}$.

\section{Characterization of MIP_MAA-co-TRIM and NIP_MAA-co-TRIM}

Characterization of MIP_MAA-co-TRIM and NIP_MAA-co-TRIM uses several instruments including Scanning Electrons Micrographs (SEM) for surface and morphological characteristics of the shape and size of the object particles, Energy Dispersive X-ray Spectroscopy (EDS) for elemental analysis, Fourier Transform Infrared (FTIR) to determine active functional groups and Thermo gravimetric Analysis (TGA) to determine the thermal stability of MIP_MAA-co-TRIM and NIP_MAA-co-TRIM. 


\section{The pH effect on MIP_MAA-co-TRIM adsorption}

The total of $20 \mathrm{mg}$ of MIP_MAA-co-TRIM was put into four vials and then added $3 \mathrm{ml}$ of $10 \mathrm{ppm} \beta$ sitosterol solution with $\mathrm{pH}$ variation of $4,5,6$, and 7 . The solution was shaken at room temperature for 6 hours and then filtered. The concentration of $\beta$-sitosterol in the filtrate was analyzed by a UV spectrophotometer at a wavelength of $202 \mathrm{~nm}$

\section{The effect of contact time on adsorption of MIP_MAA-co-TRIM}

The each of five vials was filled with $20 \mathrm{mg}$ MIP_MAA-co-TRIM then added $3 \mathrm{ml}$ of $10 \mathrm{ppm} \beta$ sitosterol solution. The adsorption process was done at different time settings for 30, 60, 120, 180, 240 minutes at room temperature and then the solution was filtered. The $\beta$-sitosterol concentration was analyzed by a UV spectrophotometer at $202 \mathrm{~nm}$ wavelength.

\section{The test of NIP and MIP adsorption ability}

The adsorption ability of MIP_MAA-co-TRIM to the target molecule was tested. By putting $20 \mathrm{mg}$ of MIP and NIP into an erlenmeyer and then added with $3 \mathrm{ml}$ of $10 \mathrm{ppm}$ $\beta$-sitosterol solution. The solution was shaken for one hour at room temperature and the filtrate was analyzed by HPLC.

\section{Result and Dicussion}

\section{Synthesis MIP and NIP}

Molecularly Imprinted Polymers (MIP_MAA) and Non Imprinted Polymers (NIP_MAA) synthesized were white powders.

\section{Characterization of MIP and NIP}

\section{FTIR analysis}

FTIR data from the NIP and MIP obtained can explain the functional groups that have an effect on polymer formation. Figure 1 shows that, the absorption peaks on the NIP_MAA-co-TRIM and MIP_MAA-coTRIM (before washing) spectrums are changing when compared to the MAA monomer spectrum. The shift of the absorption peak of the $\mathrm{OH}$ group from MAA to NIP_MAA-co-TRIM and MIP_MAA-co-TRIM (before washing) is due to the interaction of the - $\mathrm{OH}$ group of the MAA monomer with the - $\mathrm{OH}$ functional group of $\beta$-sitosterol or - $\mathrm{OH}$ group between monomers to form hydrogen bonds with wave number value as given in Table 1.

\section{Results and Discussion}

\section{Synthesis MIP and NIP}

Molecularly Imprinted Polymers (MIP_MAA) and Non Imprinted Polymers (NIP_MAA) synthesized were white powders.

\section{Characterization of MIP and NIP}

\section{FTIR analysis}

FTIR data from the NIP and MIP obtained can explain the functional groups that have an effect on polymer formation. Figure 1 shows that, the absorption peaks on the NIP_MAA-co-TRIM and MIP_MAA-coTRIM (before washing) spectrums are changing when compared to the MAA monomer spectrum. The shift of the absorption peak of the $\mathrm{OH}$ group from MAA to NIP_MAA-co-TRIM and MIP_MAA-co-TRIM (before washing) is due to the interaction of the - $\mathrm{OH}$ group of the MAA monomer with the - $\mathrm{OH}$ functional group of $\beta$-sitosterol or - $\mathrm{OH}$ group between monomers to form hydrogen bonds with wave number value as given in 
Table 1.

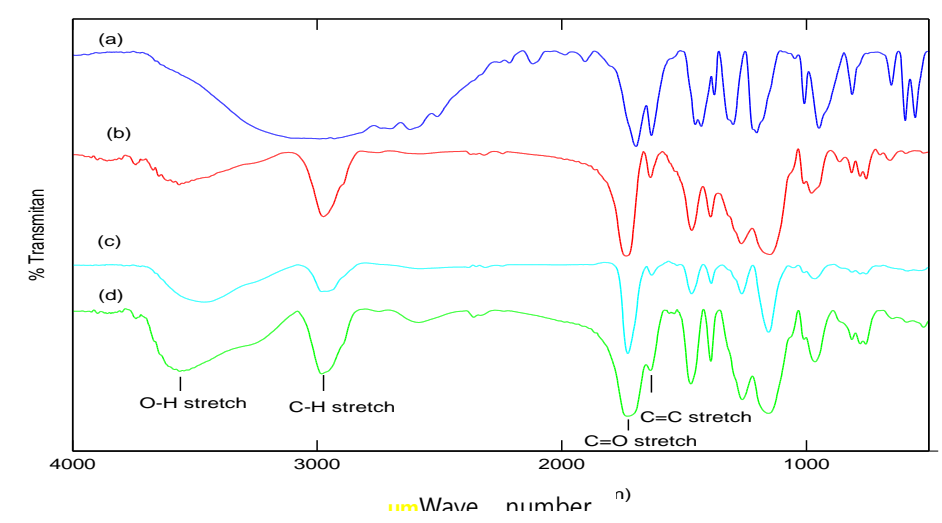

Figure 1. Spectrum of FTIR: a) MAA, b) NIP_MAA-co-TRIM, c) MIP_MAA-co-TRIM (before washed or bw) d) MIP_MAA-co-TRIM (after washing or aw)

The interaction may also occur between the $-\mathrm{C}=\mathrm{O}$ functional groups in MAA and $-\mathrm{OH}$ monomers of $\beta$ sitosterol forming a hydrogen bond causing a shift of the wave numbers on $-\mathrm{C}=\mathrm{O}$ function group in NIP_MAAco-TRIM and MIP_MAA-co-TRIM (bw).

The wave number for- $\mathrm{C}=\mathrm{C}$ functional group of MAA monomer undergoes a very small shift. However, Figure 1 shows that the intensity and the sharp of the--C=C stretching vibration of the MAA monomer reduce after the formation of NIP_MAA-co-TRIM and MIP_MAA-co-TRIM. These indicated that there were interaction between the $-\mathrm{C}=\mathrm{C}$ functional group of MAA and the cross linker.

Table 1. The Absorption of functional groups of MAA, NIP_MAA-co-TRIM, MIP_MAA-co-TRIM (bw), and MIP_MAA-co-TRIM (aw).

\begin{tabular}{|c|c|c|c|c|}
\hline \multirow{2}{*}{$\begin{array}{c}\text { Functional } \\
\text { groups }\end{array}$} & MAA & $\begin{array}{c}\text { NIP_MAA- } \\
\text { co-TRIM }\end{array}$ & $\begin{array}{c}\text { MIP_MAA-co- } \\
\text { TRIM (bw) }\end{array}$ & $\begin{array}{c}\text { MIP_MAA- } \\
\text { co-TRIM (aw) }\end{array}$ \\
\cline { 2 - 5 } & $3400-3200$ & 3564 & 3460 & 3560 \\
\hline -O-H stretching & 1695 & 1735 & 1730 & 1732 \\
\hline C=O stretching & 1633 & 1637 & 1633 & 1637 \\
\hline C=C stretching & &
\end{tabular}

The wave number of the -OH stretching vibration in MIP_MAA-co-TRIM (after washing) is greater than that in MIP_MAA-co-TRIM (bw) as a result of the breaking of the hydrogen bond between the -OH functional group in the $\beta$-sitosterol with the active site of the MIP_MAA-co-TRIM (bw) template.

\section{Binding evaluation of MIP_MAA-co-TRIM}

In MIP synthesis, non-covalent interactions developed by Mosbach and Arshadyat 1981 are most commonly used because they are simple and flexible $\mathrm{e}^{14}$. In non-covalent MIP synthesis, the interactions between template molecules and monomers are not strong enough to maintain the complex (weak intermolecular interaction). Therefore, excess monomers are required for complex stability ${ }^{5}$.The polymerization steps of MIP synthesis are the initiation, propagation and termination phase. Synthesis through the polymerization step of MIP_MAA-co-TRIM is proposed as in Figure 2.

In the pre-polymerization step (Figure 2), the monomers and molds dissolved in toluene interact in advance non-covalently by forming hydrogen bonds. The polymerization step begins with initiation by free radicals from AIBN interacting with the double bonds of TRIM to form free radicals, or interacting with the double bond of MAA to form free radicals and followed by chain extension (propagation) of the monomer or other cross linking forming new bonds. 
Termination of the chain extension (termination) occurs after two radicals of the chain extension result interact. The next step is the release of $\beta$-sitosterol in order to obtain molds with functional groups corresponding to $\beta$-sitosterol as the target molecule.

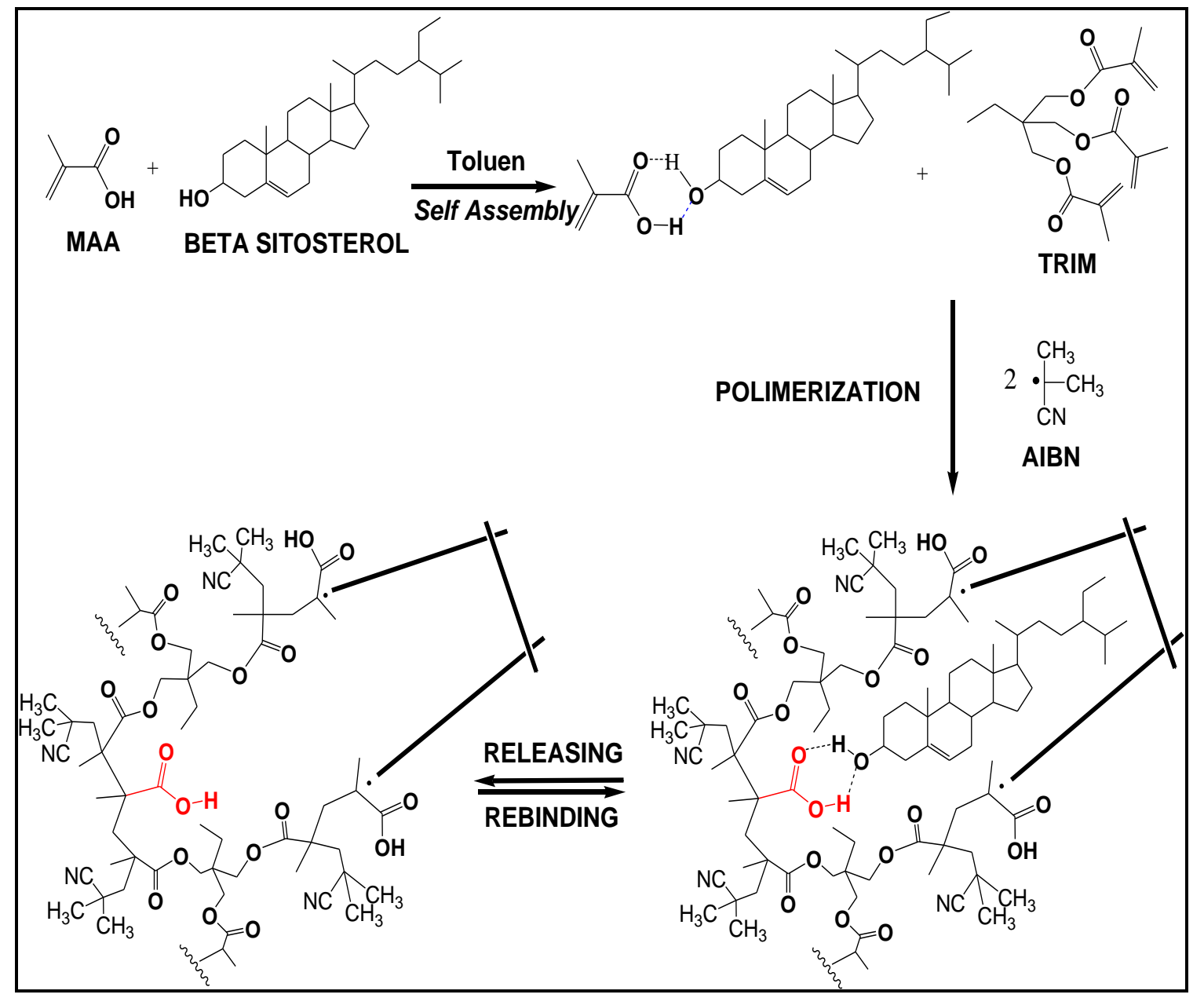

Figure 2. Proposed schemes for MIP_MAA-co-TRIM synthesis pathway

$\beta$-sitosterol may react non-covalently with the - $\mathrm{COOH}$ group of MIPs by hydrogen bonds due to the suitability of shape, size, and functional groups.

\section{Morphological Characterization of MIP and NIP}

\section{Analysis of SEM}

The result of morphological images of NIP_MAA-co-TRIM and MIP_MAA-co-TRIM using SEM can be seen in Figure 2. The surface morphology of NIP_MAA-co-TRIM is composed of an aggregate of granules with spherical form that coalesce with each other. The diameter of the granules was about $2.5 \mu \mathrm{m}$. The surface morphology of NIP_MAA-co-TRIM was similar to MIP_MAA-co-TRIM (bw), whereas surface morphology of MIP_MAA-co-TRIM (aw) appears to be composed of round grains of similar size and more porous, Indicates that the template molecule has been separated from MIP. The geometric structure of MIP_MAA-co-TRIM looks more rigid. 


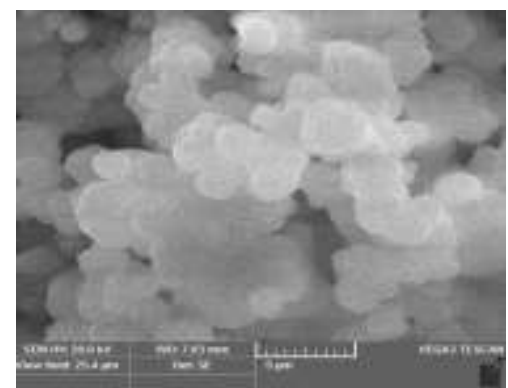

(a)

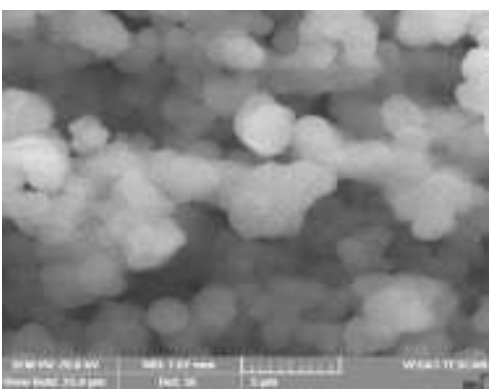

(b)

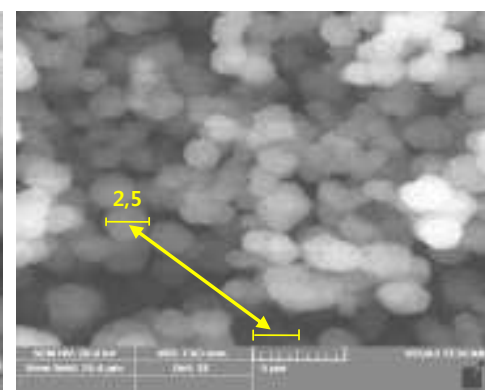

(c)

Figure 2. Morphology: a) NIP_MAA-co-TRIM, b) MIP_MAA-co-TRIM (bw), c) MIP_MAA-co-TRIM (aw)

\section{Analysis of EDS}

EDS analysis data in the Table 2 showing the composition of the main constituent elements on NIP_MAA-co-TRIM, MIP_MAA-co-TRIM (bw), MIP_MAA-co-TRIM (aw) as results of interpretation of the EDS spectrum for NIP_MAA-co-TRIM (Figure 3), MIP_MAA-co-TRIM (bw) (Figure 4) and MIP_MAA-coTRIM (Figure 5).

Table 2. EDS data of NIP, MIP_MAA-co-TRIM(bw), MIP_MAA-co-TRIM(aw), NIP_MAA-co-TRIM

\begin{tabular}{|c|c|c|c|c|c|c|}
\hline \multirow{2}{*}{ Atoms } & \multicolumn{3}{|c|}{ \% Mass } & \multicolumn{3}{c|}{ \% Atom } \\
\cline { 2 - 7 } & NIP & $\mathbf{M I P}_{(\mathrm{bw}}$ & $\mathbf{M I P}_{(\mathrm{aw}}$ & \multirow{2}{*}{ NIP } & $\mathbf{M I P}_{(\mathrm{bw}}$ & $\mathbf{M I P}_{(\mathrm{aw}}$ \\
\hline $\mathrm{C}$ & 79.04 & 80.42 & 77.31 & 83.40 & 84.55 & 81.95 \\
\hline $\mathrm{O}$ & 20.96 & 19.58 & 22.69 & 16.60 & 15.45 & 18.05 \\
\hline
\end{tabular}

bw: before washing, aw: after washing

The data show that the presence of $\beta$-sitosterol causes the number of $\mathrm{C}$ atoms to increase in MIP_MAA-co-TRIM (bw), this causes the percentage value ofC at MIP_MAA-co-TRIM (bw) is higher than in MIP_MAA-co-TRIM (aw).

The number of $\mathrm{C}$ atoms in $\beta$-sitosterol is more than that of $\mathrm{O}$, so that when $\beta$-sitosterol is still in the polymer then the character of $\mathrm{C}$ atom increases. While the $\beta$-sitosterol was released from the polymer, the character of the $\mathrm{O}$ atom increases as shown the data in Table 1. The shift of the wave number of the -OH group on the MIP_MAA-co-TRIM (TE) spectrum indicates that the hydrogen bond between the functional groups in the polymer and $\beta$-sitosterol has been broken.

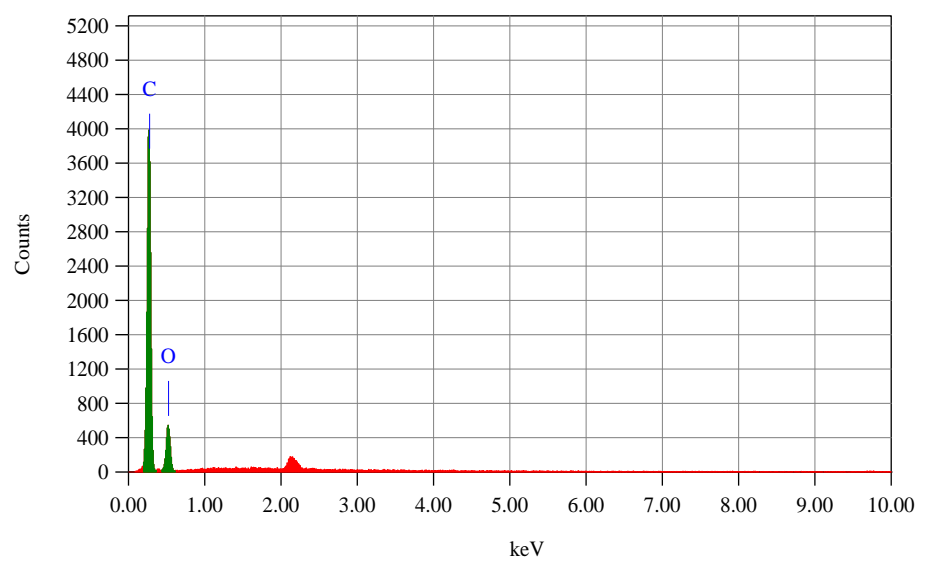

Figure 3. The EDS Spectrum of NIP_MAA-co-TRIM 


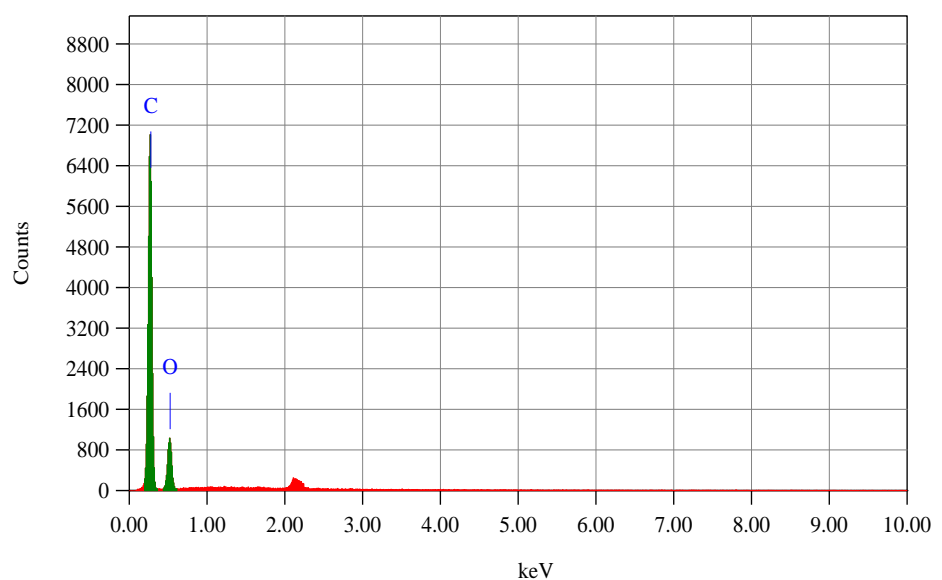

Figure 4. The EDS Spectrum of MIP_MAA-co-TRIM (before washing)

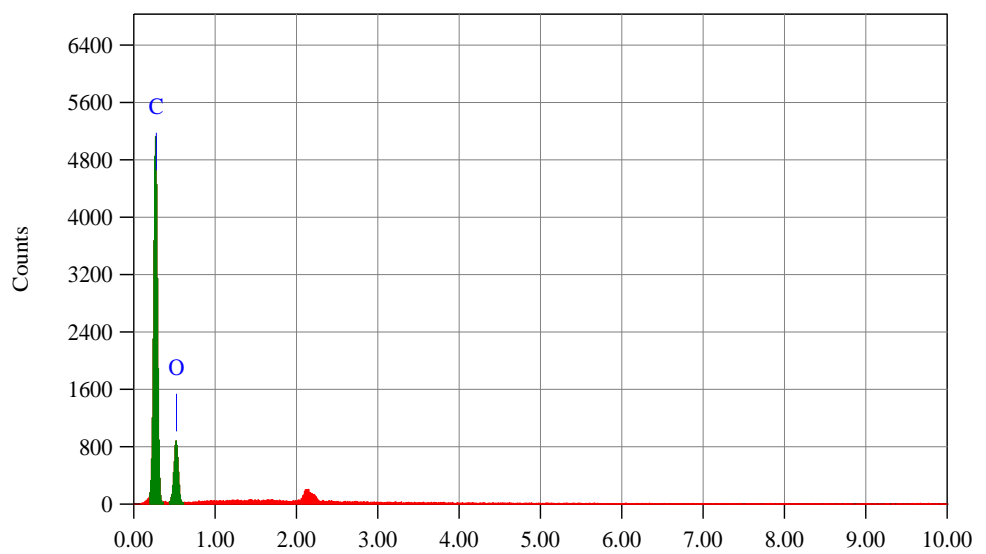

Figure 5. The EDS Spectrum of MilP_MAA-co-TRIM (after washed)

\section{Analysis of TGA}

The result of TGA analysis can be seen in Figure 6.NIP_MAA-co-TRIM thermogram has a very similar pattern to that of MIP_MAA-co-TRIM (aw). This happens because after washing, $\beta$-sitosterol is no longer exist in MIP_MAA-co-TRIM (aw) MIP_MAA-co-TRIM (bw) is different because it still contains $\beta$ sitosterol.

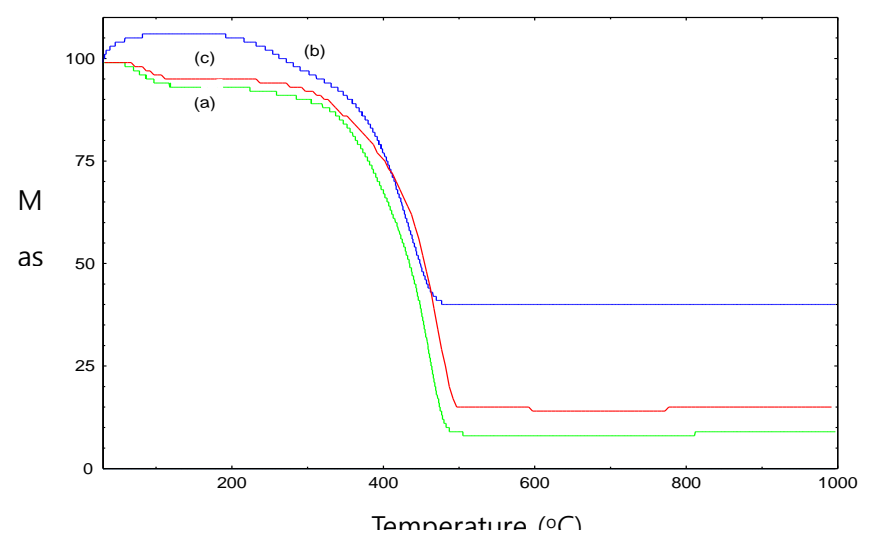

Figure 6. Thermograms of (a). NIP_MAA-co-TRIM, (b) MIP_MAA-co-TRIM (bw) and (c). MIP_MAA-co-TRIM (aw) 
Based on the data derivative of TGA (DTG,) NIP and MIP undergoing several time mass losses. The lost initial mass was thought to be the mass of water. Massive mass losses occur at temperatures around $402.6^{\circ} \mathrm{C}$ to $492.6^{\circ} \mathrm{C}$ for NIP_MAA-co-TRIM, temperatures of about $355.93^{\circ} \mathrm{C}$ to about $479.93^{\circ} \mathrm{C}$ for MIP_MAA-co-TRIM (bw), and above temperature $352.91^{\circ} \mathrm{C}$ to $482.91^{\circ} \mathrm{C}$ for MIP_MAA-co-TRIM (aw). At this temperature, suspected degradation occurs in MIP so that $\mathrm{CO}$ and $\mathrm{CO}_{2}$ are formed. Higher temperatures cause breaking of the bond between the monomer and the cross-linker and leaving carbon. NIP and MIP began to degrade at high temperatures, indicating that MIP_MAA-co-TRIM was a stable polymer at high temperature.

\section{Analysis of TEM}

The TEM analysis results show morphological differences between MIP_MAA-co-TRIM and NIP_MAA-co-TRIM. The MIP_MAA-co-TRIM forms an aggregate composed of cavities with a pore size on a surface of about $50 \mathrm{~nm}$ and spherical in shape. The pores distributed on the surface can be seen in Figure 7. The cavities in MIP_MAA-co-TRIM are the spaces formed after the template removal. While NIP_MAA-co-TRIM also forms an aggregate without cavities and pores as it is made without using $\beta$-sitosterol as a cavity-printing molecule.

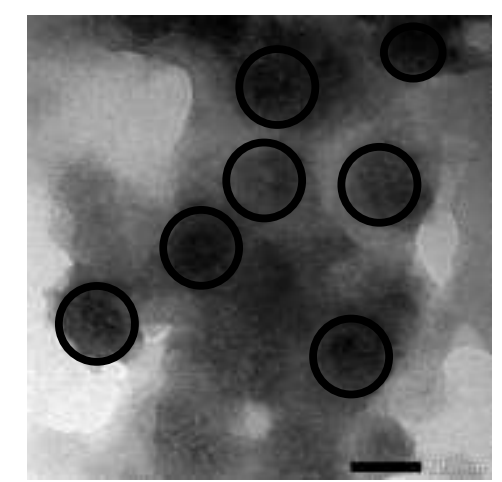

A)

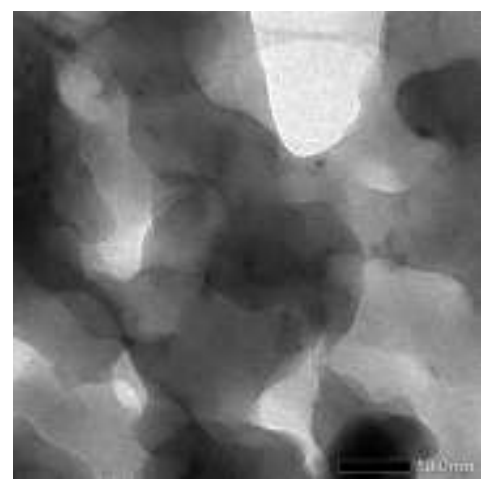

B)

Figure 7. Morphologies of: A) MIP_MAA-co-TRIM and B) NIP_MAA-co-TRIM

\section{The adsorption ability of MIP}

The adsorption ability test was performed to determine the ability of NIP_MAA-co-TRIM and MIP_MAA-co-TRIM in adsorbing $\beta$-sitosterol compounds. The amount of $\beta$-sitosterol in the adsorption process by MIP can be seen in Table 1 .

Table 1. The amount of $\beta$-sitosterol adsorbed by NIP_MAA-co-TRIM and MIP_MAA-co-TRIM

\begin{tabular}{|c|c|}
\hline Kinds of polymer & $\begin{array}{c}\text { The amount of } \boldsymbol{\beta} \text {-sitosterol adsorbed } \\
(\mathbf{q})(\mathbf{m g} / \mathbf{g})\end{array}$ \\
\hline $\begin{array}{c}\text { NIP_MAA-co- } \\
\text { TRIM }\end{array}$ & 0.05 \\
\hline $\begin{array}{c}\text { MIP_MAA-co- } \\
\text { TRIM }\end{array}$ & 0.75 \\
\hline
\end{tabular}

It is clear that MIP_MAA-co-TRIM can adsorb $\beta$-sitosterol better than NIP_MAA-co-TRIM. Because of the functional groups of $\mathrm{O}-\mathrm{H}$ and $\mathrm{C}=\mathrm{O}$ on MIP surfaces and cavities that have an affinity for the $\mathrm{OH}$ functional group of $\beta$-sitosterol to form hydrogen bonds. While in NIP, the functional groups of $\mathrm{O}-\mathrm{H}$ and $\mathrm{C}=\mathrm{O}$ are present only on the surface.

\section{Effect of pH on MIP_MAA-co-TRIM adsorption}

The optimum $\mathrm{pH}$ was determined by studying the effect of $\mathrm{pH}$ on MIP_MAA-co-TRIM adsorption capability. The effect of $\mathrm{pH}$ can be seen in Figure 8. 


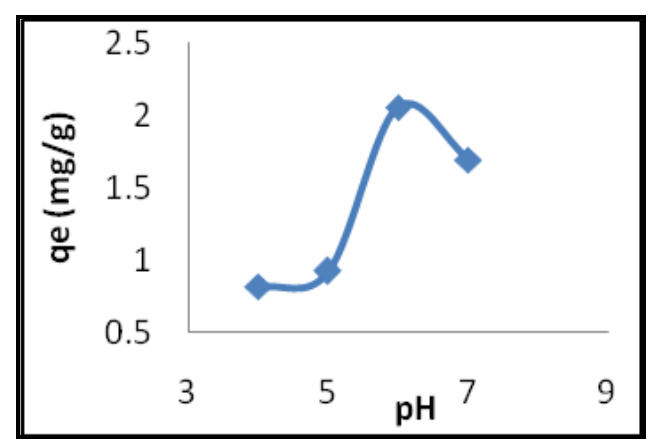

Figure 8. The pH optimum curve of MIP_MAA-co-TRIM

Adsorption of $\beta$-sitosterol on MIP-MAA-co-TRIM are to which showed optimum at pH 6 and decreased at higher $\mathrm{pH}$. The adsorption capacity of MIP is low at $\mathrm{pH}$ below optimum because the concentration of $\mathrm{H}^{+}$ions in the solution is high, resulting in protonation of the functional group on the adsorbent and the hydroxyl functional group of $\beta$-sitosterol. As a result, there is an electrostatic repulsion between the adsorbent and the target molecule causing low levels of $\beta$-sitosterol adsorbed by MIP. The formation of hydrogen bonds increases between the hydroxyl functional groups in the hydroxyl group of MAA monomer at optimum $\mathrm{pH}$ because the concentration of $\mathrm{H}^{+}$ions in the solution begins to decrease. When the $\mathrm{pH}$ increases to $\mathrm{pH} 7, \beta$ sitosterol is more neutral, so that the hydrogen bond interaction occurs with the active polymer.

\section{The Influence of Time}

Figure 9 shows that the adsorption of $\beta$-sitosterol on MIP_MAA-co-TRIM is different at all times. The adsorption increases with time until equilibrium is reached at 60 minutes, but decreases with increasing time. This happens because the surface of the adsorbent has been saturated by $\beta$-sitosterol.

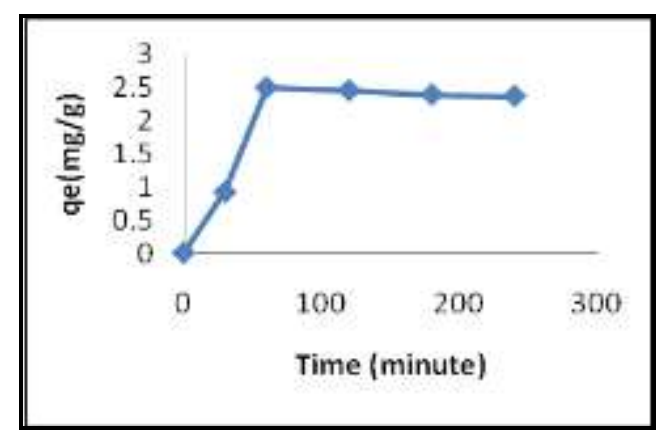

Figure 9. The amount of $\beta$-sitosterol adsorbed on MIP_MAA-co-TRIM as a function of contact times

Figure 10 shows the kinetic curves of pseudo-first order and pseudo-second order of $\beta$-sitosterol adsorption on MIP_MAA-co-TRIM.

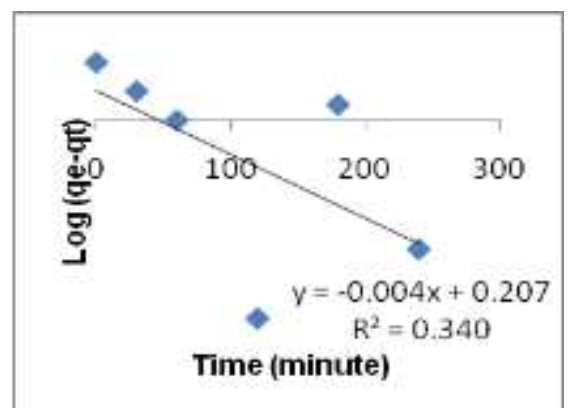

(a)

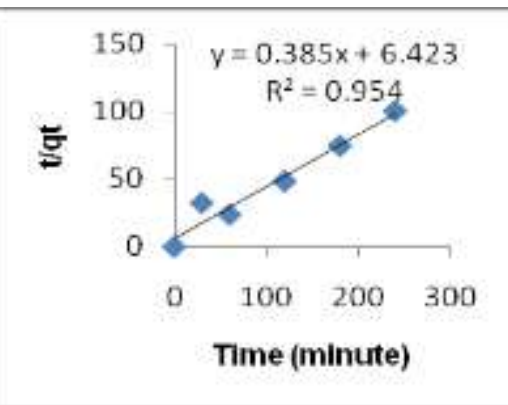

(b)

Figure 10. The Kinetic curves of (a) pseudo-order first and (b) pseudo-order second for $\beta$-sitosterol adsorption by MIP_MAA-co-TRIM 
The adsorption kinetic data for MIP_MAA-co-TRIM were also analyzed using the pseudo-order first and pseudo-order second equations. The values of $\mathrm{R}_{2}, \mathrm{k}_{1}, \mathrm{k}_{2}, q_{\mathrm{e}}$ of calculation and $q_{\mathrm{e}}$ of experimental results are shown in Table 2

Table 2. The Kinetic parameter of MIP_MAA-co-TRIM

\begin{tabular}{|c|c|c|c|c|c|}
\hline $\begin{array}{c}\text { The kinetic } \\
\text { adsorption }\end{array}$ & $\mathbf{k}_{\mathbf{1}}$ & $\mathbf{k}_{\mathbf{2}}$ & $\begin{array}{c}\boldsymbol{q}_{e} \\
\text { calculation } \\
(\mathbf{m g} / \mathbf{g})\end{array}$ & $\begin{array}{c}\boldsymbol{q}_{e} \\
\mathbf{e x p e r i m e n t} \\
(\mathbf{m g} / \mathbf{g})\end{array}$ & $\mathbf{R}^{\mathbf{2}}$ \\
\hline $\begin{array}{c}\text { The pseudo- } \\
\text { first order }\end{array}$ & 0.0092 & - & 1.61 & 2.51 & 0.340 \\
\hline $\begin{array}{c}\text { The pseudo- } \\
\text { second order }\end{array}$ & - & 0.0231 & 2.60 & 2.51 & 0.954 \\
\hline
\end{tabular}

The data show that the pseudo-second order curves gives a better correlation of adsorption data than the pseudo-first order curve and the least square value $\left(\mathrm{R}^{2}\right)$ for the pseudo-second order model approaches 1 , whereas that of the pseudo-first order model is not close to 1 . The amount adsorbed $\left(\mathrm{q}_{\mathrm{e}}\right)$ obtained from calculation for the pseudo-second order is closer to the experimental value. Therefore, it can be concluded that the pseudo-second order is more appropriate as the adsorption kinetics model for MIP_MAA-co-TRIM than the pseudo-first order.

\section{Conclusion}

Rigid white solids of NIP and MIP have been produced by the bulk polymerization through free radical polymerization reactions. Functional groups of $\mathrm{OH}, \mathrm{C}=\mathrm{O}$ and $\mathrm{C}=\mathrm{C}$ play a role in the formation of NIP_MAAco-TRIM and MIP_MAA-co-TRIM. MIP_MAA-co-TRIM can better adsorb $\beta$-sitosterol than NIP_MAA-coTRIM. The surface morphology of MIP_MAA-co-TRIM was more rough and porous than NIP_MAA-coTRIM. The optimum condition of MIP to adsorb $\beta$-sitosterol was at the $\mathrm{pH}$ of 6 and the time of $60 \mathrm{~min}$. The adsorption of $\beta$-sitosterol on MIP_MAA-co-TRIM fitted the pseudo second order model.

\section{Acknowledgment}

Thanks to The Directorate of Higher Education-Indonesia forthe financial support and also to The University of Hasanuddin for supporting facilities in this research.

\section{References}

1. Beltran, A.F., Borrull, P.A.G.; Cormack, R.M., Marce. Molecularly-Imprinted Polymers: useful sorbents for selective extractions. Trends in Analytical Chemistry. 2010, 29, No. 11

2. Blahova E., LehotayJ., Skac`a'ni I. The Use of Molecularly Imprinted Polymer for Selective Extraction of (1)-Catechin. Journal of Liquid Chromatography \& Related Technologies. 2004, 27, No. 17, pp. 2715-2731

3. Chen, L., Jia, X., Lu, Q., Peng, Y., Du, S., Chen, Q. Highly Efficient and Selective Enrichment of PuerarinFrom Radix Puerarieae by Molecularly Imprinted Solid-Phase Extraction. Sep. Pur. Tech. 2010, 71: 324-330

4. Fauziah, S., Taba, P.; Amran, M.B., Budi, P., Soekamto, N.H. Synthesis, Characterization, and Optimization of $\beta$-sitosterol Imprinted Polymers using TFMAA as functional monomer. Int. J. of Applied Chemistry. 2015, 11,487-495

5. Holland, N. An Investigation into the Role of Morphology on the Performance of Molecularly Imprinted Polymers, Separation Science Research Group. Waterford Institute of Technology, Ireland. 2008, 1-267

6. Jinfang W., Peter A.G.C., David C.S., Ezat K. Synthesis and Characterization of micrometer-sized molecularly imprinted spherical polymer particulates prepared via precipitation polymerization. Pure Appl. Chem. 2007, 79, No.9, pp. 1505-1519 
7. Kim, H. Spivak, D.A. New insight into modeling non-covalently imprinted polymers J. Am. Chem. Soc. 2003, 125, 11269-11275

8. Mayes, A.G.., Whitcombe, M. Synthetic strategies for the generation of Molecularly Imprinted Polymers. J. Advanced Drug Delivery Review. 2005, 57, 1742-1778

9. Sellergen, B., Allender, C. Molecularly imprinted polymers : A bridge to advance drug delivery. Advance drug delivery reviews. 2005, 57: 1733-1741

10. Soekamto, N.H., Fauziah, St., Taba, P., Amran, M.B. Adsorption of $\beta$-sitosterol on molecularly imprinted polymer. IOP Conf. Series: Materials Science and Engineering. 2017, 188, 012048

11. Song, X., Li, J., Xu, S., Ying, Rongjian, Ma, J., Liao, C., Liu, D., Yu, J., Chen, L. Determination of 16 Polycyclic Aromatic Hydrocarbons in seawater using Molecularly Imprinted Solid Phase Extraction Coupled with GC-MS. Talanta. 2012, $99:$ 75-82

12. Surikumaran, H., Mohammad, S., and Sari, N.M. Molecular Imprinted polymer of Methacrylic Acid Functionalized $\beta$-Cyclodextrin for Selective Removal of 2,4-Dichlorophenol. Int. J. Mol. Sci. 2014,15 : 6111-6136

13. Tao Z., Changhwan Y., Kyungho R. Solid-phase Extraction of â-sitosterol from Oldenlandia diffusa Using Molecular Imprinting Polymer. Chin. J. Chem., 2011, 29, 1246-1250.

14. Walsh, R. Thesis. Pharmaceutical and Molecular Biotechnology Research Centre, Waterford Institute of Technology. 2010

15. Xu W.L., Huang Y.B., Qian J.H., Sha O., Wang Y.Q, Separation and purification of stigmasterol and beta-sitosterol from phytosterol mixtures by solvent crystallization method. Separation and Purification Technology. 2005, 41,173-178

16. Yan, H., and Row, K.H. Characteristic and Synthetic Approach of Molecularly Imprinted Polymer. Int. J. Mol. Sci., 2006,7: 155-178.

17. Yavuz H., Karakoc V., Turkmen D., Say R., Denizli A. Synthesis of cholesterol imprinted polymeric particles. Int. J. Bio. Macromolecules, 2007, 41: 8-15

18. Zhu Q., Tang J., Dai J.;Gu X., Chen S. Synthesis and Characteristics of Imprinted 17-b-estradiol Microparticle and Nanoparticle with TFMAA as Functional Monomer. J. Applied Polymer Sci., 2007, 104 : 1551-1558. 